\title{
EXCITATION DYNAMICS OF TWO LEVEL QUANTUM SYSTEMS COUPLED TO MORSE VIBRATIONS
}

\author{
D. Abramavičius and T. Marčiulionis \\ Institute of Chemical Physics, Vilnius University, Sauletekio 9, 10222 Vilnius, Lithuania \\ Email: darius.abramavicius@ff.vu.lt
}

Received 5 November 2018; accepted 2 January 2019

\begin{abstract}
Electronic excited states of a molecular aggregate coupled to Morse vibrations are analysed by a nonperturbative time dependent variational approach. General equations of motion for an electronically excited state are derived for electronic amplitudes, nuclear displacements and squeezing of the nuclear wave packets. Numerical simulations demonstrate that anharmonicities of vibrations lead to short-term irreversible dynamics, extra localization and transformation of stationary lowest-energy states.
\end{abstract}

Keywords: excitons, polarons, nonlinear vibrations, molecular aggregates

\section{Introduction}

Functional molecular aggregates have been extensively studied as possible candidates to replace inorganic atomic semiconductors that have relatively high production costs [1, 2]. Molecular organic light emitters and organic solar cells are now successfully employed in industry. Organic molecular aggregates are also the main building blocks in energy production in natural photosynthesis [3, 4 .

Modelling of such systems usually relies on a tight-binding or Frenkel exciton models additionally coupled to harmonic vibrations, representing molecular and phonon vibrations [5, 6]. Such formulation allows recovery of exciton relaxation dynamics, charge separation and polaron formation as well as charge transport in a broad range of problems. Apart from dynamics, it should be noted that the bath of harmonic vibrations allows describing realistic spectral line shapes in molecular spectroscopy [7]. The fast bath fluctuations lead to homogeneous broadenings, slow or static deviations cause inhomogeneous broadenings, specific peaks in phonon spectral densities show the vibra- tional progressions observed in molecular systems especially at low temperatures [8].

However, the unexpected outcome of the model is an infinitely narrow lowest-energy zero-phonon electronic band. It has no contribution to spectral broadening at low temperature when realistic phonon spectral density $\left(C^{\prime \prime}(\omega) \propto \omega^{3}\right.$ at $\left.\omega \rightarrow 0\right)$ is taken into account [9]. Even at higher temperatures the spectral broadening of the zero phonon line (ZPL) comes only from the 'finite lifetime' broadening effects. Additionally, the linear models lead to resistance to thermal conduction in the bath and essentially to blocking of thermal equilibration [10].

It has been shown that the ZPL line width becomes finite when a nonlinear electron-vibrational coupling is included in the model [11]. This property is primarily due to different vibrational frequency in electronic ground and excited states. However, the quadratic coupling is only the second term in the series expansion in the electron-phonon coupling potential. The realistic potentials are neither harmonic nor the interaction functions are simple power functions [12-14], and that may lead to novel physics and spectroscopy. 
In this paper we take a more realistic Morse type molecular vibrational potential into account and derive equations of motion of the electronic system coupled to such vibrations by using a nonperturbative approach developed earlier [15]. We find that interaction potentials are essentially exponential functions of vibrational coordinates. The net result is that the anharmonicities cause an additional localization of electronic excitations and different symmetry of relaxed electronic excitations.

\section{Theory}

\subsection{Model}

We start from the standard model of Frenkel exciton coupled to molecular vibrations. The electronic ground state is taken as a reference, where all degrees of freedom are independent. In the electronic excited state the equilibrium position of a vibration becomes shifted, so the energy of the excited state becomes dependent on the coordinate of the vibration. Such a system is described by the following Hamiltonian:

$$
\begin{aligned}
\hat{H} & =\sum_{m n} J_{m m}|m\rangle\langle n|+\sum_{i} \hat{K}\left(\hat{x}_{i}\right)+V_{i}\left(\hat{x}_{i}\right) \\
& +\sum_{n} \sum_{i}|n\rangle\langle n|\left(V_{i}\left(\hat{x}_{i}-d_{n i}\right)-V_{i}\left(\hat{x}_{i}\right)\right) .
\end{aligned}
$$

Here $\hat{x}_{\mathrm{i}}$ is the coordinate operator of vibrational mode $i, V_{i}(x)$ is its potential energy function. Assuming a unit mass case, the kinetic energy operator takes a simple form $\hat{K}\left(\hat{x}_{i}\right)=\hat{x}_{i}{ }_{i} / 2, \hat{x}_{i}$ being equivalent to the momentum operator. The matrix $J_{m n}$ is the electronic coupling matrix for orthogonal excited states $|m\rangle,|n\rangle$; the electronic ground state $|0\rangle$ forms a separate manifold with zero electronic energy.

The traditional harmonic model is defined by $V_{i}\left(x_{i}\right)=\omega_{i}^{2} x_{i}^{2} / 2$ for all modes [16]. $\omega_{i}$ is the harmonic frequency. It leads to the linear coupling scenario, $V_{i}\left(x_{i}-d_{n i}\right)-V_{i}\left(x_{i}\right)=\Lambda_{n i}-g_{n i} x_{i}$, where $\Lambda_{n i}=\omega_{i}^{2} d_{n i}^{2} / 2$ is the reorganization energy with the electronicvibrational coupling constant $g_{n i}=\omega_{i}^{2} d_{n i}$.

In the present paper we assume that all vibrational modes are characterized by the anharmonic Morse potential defined by

$$
V_{i}\left(x_{i}\right)=\mathcal{D}_{i}\left(1-\exp \left(-\alpha_{i} x_{i}\right)\right)^{2} .
$$

Here $\mathcal{D}_{i}$ is the classical dissociation energy and $\alpha_{i}$ defines the width of the potential. The potential minimum is at $x_{i}=0$ while the curvature at the minimum corresponds to the frequency $\omega_{i}=\sqrt{2 \mathcal{D}_{i} \alpha_{i}^{2}}$.

The Morse potential yields an exponential type of the electron-vibrational interaction

$$
V_{i}\left(x_{i}-d_{n i}\right)-V_{i}\left(x_{i}\right)=\mathcal{D}_{i}\left(\left(2+s_{n i}\right) e^{-2 \alpha_{i} x_{i}}-2 e^{-\alpha_{i} x_{i}}\right),
$$

where $s_{n i}=\exp \left(\alpha_{i} d_{n i}\right)-1$. The corresponding reorganization energy $\Lambda_{n i}=\mathcal{D}_{i} s_{n i}^{2}$ : Notice that for a narrow potential when $\alpha_{i} x_{i} \ll 1$, we obtain $s_{\text {in }} \approx \alpha_{i} d_{\text {in }}$ and the Morse model reduces to the harmonic model with the leading linear coupling term.

\subsection{Wave packet Ansatz}

It is possible to model the explicit quantum mechanical propagation of a system wave vector according to the Schrödinger equation for such system numerically, however, that is computationally inefficient as the number of degrees of freedom grows up exponentially with the number of vibrational modes. Instead, for a manifold of singly excited electronic states we choose to use the time dependent variational approach based on the Ansatz of squeezed coherent states [17. 18, 15,:

$$
|\psi\rangle=\sum_{n} a_{n}|n\rangle \prod_{i} \hat{D}_{i}\left(\lambda_{i}\right) \hat{S}_{i}\left(\zeta_{i}\right)\left|0_{v}\right\rangle .
$$

Here the parameterized system wave packet is constructed from a superposition of electronic amplitudes of all relevant excitations. The vibrational manifold is constructed from the vibrational zero-quanta state, $\left|0_{v}\right\rangle$, by acting with the squeeze, $\hat{S}_{i}\left(\zeta_{i}\right)=\exp \left(\left(\zeta_{i}^{*} \hat{b}_{i}^{2}-\zeta_{i} \hat{b}_{i}^{\dagger}{ }^{2}\right) / 2\right)$, and displacement, $\hat{D}_{i}\left(\lambda_{i}\right)=$ $\exp \left(\lambda_{i} \hat{b}_{i}^{\dagger}-\lambda_{I}^{*} \hat{b}_{I}\right)$ operators. The main properties of the operators are described in Appendix A. $a_{n}, \zeta_{i}$, $\lambda_{i}$ are the time-dependent parameters of the wave packet, while $\hat{b}_{i}$ and $\hat{b}_{i}^{\dagger}$ are the standard bosonic ladder operators related to the coordinate and momentum by standard relations:

$$
\begin{aligned}
& \hat{x}_{i}=\frac{1}{\sqrt{2 \omega_{i}}}\left(\hat{b}_{i}^{\dagger}+\hat{b}_{i}\right), \\
& \hat{\dot{x}}_{i}=\mathrm{i} \sqrt{\frac{\omega_{i}}{2}}\left(\hat{b}_{i}^{\dagger}-\hat{b}_{i}\right) .
\end{aligned}
$$


All parameters are complex-valued, however, squeezing will be more convenient to represent in terms of the real-valued amplitude and phase $\zeta_{i}=r_{i} \exp \left(\mathrm{i} \theta_{i}\right)$.

The time evolution of the wavefunction will be completely defined when we get equations of motion for the parameters. For this purpose we use the Dirac-Frenkel variational procedure. It is based on search for the extremum of the system quantum mechanical Lagrangian defined by

$$
\mathcal{L}=\frac{\mathrm{i}}{2}\left(\left\langle\psi \mid \frac{\mathrm{d}}{\mathrm{d} t} \psi\right\rangle-\left\langle\frac{\mathrm{d}}{\mathrm{d} t} \psi \mid \psi\right\rangle\right)-\langle\psi|\hat{H}| \psi\rangle .
$$

The equations of motion are derived from the Lagrangian extreme condition

$$
\frac{d}{\mathrm{~d} t} \frac{\partial \mathcal{L}}{\partial \dot{z}}-\frac{\partial \mathcal{L}}{\partial z}=0
$$

where $z$ is an arbitrary independent system parameter.

It should be noted that not all parameters of the Ansatz are independent. In particular, the time evolution of the wavefunction keeps the total amplitude of the wavefunction constant:

$$
\sum_{n}\left|a_{n}\right|^{2}=1
$$

So the norm of a single $a_{n}$ becomes dependent on all others. However, we treat all parameters as independent, while keep the normalization condition in mind for additional simplifications.

\subsection{Total energy and Lagrangian of the system}

Dynamical equations of motion according to the extreme of the Lagrangian yield the equations of motion that conserve the wavefunction norm and the total energy of the system. The total energy enters the Lagrangian, also, it is an important additional parameter to inspect if we search for the lowest energy state. As the wavefunction is defined, the energy can be easily calculated as the expectation value of the Hamiltonian

$$
\begin{aligned}
& \langle\psi|\hat{H}| \psi\rangle=\sum_{m n} J_{m n} a_{m}^{*} a_{n}+\sum_{i} \frac{\omega_{i}}{4}\left(\cosh \left(2 r_{i}\right)\right. \\
& \left.+\sinh \left(2 r_{i}\right) \cos \left(\theta_{i}\right)+4 \lambda_{i}^{(\mathrm{i}) 2}\right)
\end{aligned}
$$

$$
\begin{aligned}
& +\sum_{i} \mathcal{D}_{i}\left(1-2\left(1+\sum_{n} a_{n}^{*} a_{n} s_{i n}\right) f_{1}\left(\lambda_{i}^{(\mathrm{r})}\right) f_{2}\left(\zeta_{i}\right)\right) \\
& +\sum_{i} \mathcal{D}_{i}\left(1+\sum a_{n}^{*} a_{n} s_{i n}\left(2+s_{i n}\right)\right) f_{1}^{2}\left(\lambda_{i}^{(r)}\right) f_{2}^{4}\left(\zeta_{i}\right),
\end{aligned}
$$

where we separate $\lambda_{i}=\lambda_{i}^{(\mathrm{r})}+i \lambda_{i}^{(\mathrm{i})}$. Functions $f_{1}$ and $f_{2}$ are defined in Appendix B. The remaining, kinetic, part of the Lagrangian is essentially most complicated requiring normal ordering of all operators. Its calculation is also described in Appendix B. The full Lagrangian in terms of variables $a_{i}$, $\lambda_{i}, r_{i}$ and $\theta_{i}$ is finally given by

$$
\begin{aligned}
& \mathcal{L}=\frac{1}{2} \sum_{i}\left(\mathrm{i} \lambda_{i}^{*} \dot{\lambda}_{i}-\mathrm{i} \dot{\lambda}_{i}^{*} \lambda_{i}-\dot{\theta_{i}} \sinh ^{2}\left(r_{i}\right)\right) \\
& +\frac{\mathrm{i}}{2} \sum_{n}\left(a_{n}^{*} \dot{a}_{n}-a_{n} \dot{a}_{n}^{*}\right)-\langle\psi|\hat{H}| \psi\rangle .
\end{aligned}
$$

\section{Equations of motion of the parameters}

\subsection{Electronic excited state}

Equations of motion are obtained by a variational procedure and reffect the extremal condition of the Lagrangian. Using Appendices B and C the final set of equations can be derived in the following form:

$$
\begin{aligned}
& \dot{a}_{n}(t)=-\mathrm{i} \sum_{m} J_{n m} a_{m}-\mathrm{i} a_{n} \sum_{i} \frac{\omega_{i}^{2} s_{i n}}{2 \alpha_{i}^{2}} f_{1}\left(\lambda_{i}\right) f_{2}\left(\zeta_{i}\right) \times \\
& \times\left(\left(2+s_{i n}\right) f_{1}\left(\lambda_{i}\right) f_{2}^{3}\left(\zeta_{i}\right)-2\right) \\
& \frac{\dot{\lambda}_{i}}{\omega_{i}}=\lambda_{i}^{(\mathrm{i})}+\mathrm{i} \frac{f_{1}\left(\lambda_{i}\right) f_{2}\left(\zeta_{i}\right)}{\alpha_{i} \sqrt{2 \omega_{i}}} \times \\
& \times\left(f_{1}\left(\lambda_{i}\right) f_{2}^{3}\left(\zeta_{i}\right)-1+\sum_{n}\left|a_{n}\right|^{2} s_{i n}\left(\left(2+s_{i n}\right) f_{1}\left(\lambda_{i}\right) f_{2}^{3}\left(\zeta_{i}\right)-1\right)\right), \\
& \frac{2 \dot{r}_{i}}{\omega_{i}}=-\sin \left(\theta_{i}\right)+\sin \left(\theta_{i}\right) f_{1}\left(\lambda_{i}\right) f_{2}\left(\zeta_{i}\right) \times \\
& \times\left(2 f_{1}\left(\lambda_{i}\right) f_{2}^{3}\left(\zeta_{i}\right)-1+\sum_{n}\left|a_{n}\right|^{2} s_{i n}\left(2\left(2+s_{i n}\right) f_{1}\left(\lambda_{i}\right) f_{2}^{3}\left(\zeta_{i}\right)-1\right)\right) \\
& \frac{\dot{\theta_{i}}}{\omega_{i}}=-\left(1+\operatorname{coth}\left(2 r_{i}\right) \cos \left(\theta_{i}\right)\right) \\
& -\left(1+\operatorname{coth}\left(2 r_{i}\right) \cos \left(\theta_{i}\right)\right) f_{1}\left(\lambda_{i}\right) f_{2}\left(\zeta_{i}\right) \times \\
& \times\left(2 f_{1}\left(\lambda_{i}\right) f_{2}^{3}\left(\zeta_{i}\right)-1+\sum_{n}\left|a_{n}\right|^{2} s_{i n}\left(2\left(2+s_{i n}\right) f_{1}\left(\lambda_{i}\right) f_{2}^{3}\left(\zeta_{i}\right)-1\right)\right) .
\end{aligned}
$$


These equations can be used to construct, e.g. a molecular system coupled to the dissipative anharmonic environment.

Further, we consider an aggregate where we have only one intramolecular vibrational mode per site which is coupled only to its own site. Moreover, we assume that molecular oscillators have identical vibrational parameters so we take the unit frequency case, i.e. $\omega_{i}=1$, and essentially all parameters become with respect to this unit frequency dimensionless. In this case the parameter $\alpha_{i}=1 / \sqrt{2 \mathcal{D}_{i}}$ directly relates to the anharmonicity and ionization energy. After some simplifications we then get a slightly simpler set of equations:

$$
\begin{aligned}
& \dot{a}_{n}(t)=-\mathrm{i} \sum_{m} J_{n m} a_{m}-\mathrm{i} a_{n} \frac{s}{a^{2}} f_{1}\left(\lambda_{n}\right) f_{2}\left(\zeta_{n}\right) \times \\
& \times\left(\left(1+\frac{1}{2} s\right) f_{1}\left(\lambda_{n}\right) f_{2}^{3}\left(\zeta_{n}\right)-1\right), \\
& \dot{\lambda}_{n}=\lambda_{i}^{(\mathrm{i})}+\mathrm{i}\left(1+\left.\left|a_{n}\right|\right|^{2} s\right) \frac{f_{1}\left(\lambda_{n}\right) f_{2}\left(\zeta_{n}\right)}{\alpha \sqrt{2}} \times \\
& \times\left(\frac{1+\left|a_{n}\right|^{2} s(2+s)}{1+\left|a_{n}\right|^{2} s} f_{1}\left(\lambda_{n}\right) f_{2}^{3}\left(\zeta_{n}\right)-1\right), \\
& 2 \dot{r}_{n}=-\sin \left(\theta_{n}\right)+\sin \left(\theta_{n}\right)\left(1+\left|a_{n}\right|^{2} s\right) f_{1}\left(\lambda_{n}\right) f_{2}\left(\zeta_{n}\right) \times \\
& \times\left(2 \frac{1+\left|a_{n}\right|^{2} s(2+s)}{1+\left|a_{n}\right|^{2} s} f_{1}\left(\lambda_{n}\right) f_{2}^{3}\left(\zeta_{n}\right)-1\right), \\
& \dot{\theta}_{n}=-\left(1+\operatorname{coth}\left(2 r_{n}\right) \cos \left(\theta_{n}\right)\right)-\left(1+\operatorname{coth}\left(2 r_{n}\right) \cos \left(\theta_{n}\right)\right) \times \\
& \times\left(1+\left|a_{n}\right|^{2} s\right) f_{1}\left(\lambda_{n}\right) f_{2}\left(\zeta_{n}\right) \times \\
& \times\left(2 \frac{1+\left|a_{n}\right|^{2} s(2+s)}{1+\left|a_{n}\right|^{2} s} f_{1}\left(\lambda_{n}\right) f_{2}^{3}\left(\zeta_{n}\right)-1\right) .
\end{aligned}
$$

As in our previous study [15], equations of motion contain a problematic point $r_{i}=0$, where $\operatorname{coth}\left(2 r_{i}\right) \rightarrow \infty$. To avoid this point we replace

$$
\operatorname{coth}\left(2 r_{i}\right) \rightarrow \frac{\tanh \left(2 r_{i}\right)}{\tanh ^{2}\left(2 r_{i}\right)+\eta^{2}}
$$

where a positive real $\eta \ll 1$. In this case the point $r_{i}=0$ is avoided and the equations behave properly. In numerical simulations we use $\eta^{2}=10^{-7}$.

It can be easily shown that the equations of motion conserve the total probability. The condition $\Sigma_{n}\left|a_{n}\right|^{2}=1$ implies that

$$
\sum_{n} a_{n}^{*} \dot{a}_{n}+\text { c.c. }=0 \text {. }
$$

Then from the first equation we have

$$
\begin{aligned}
& \dot{a}_{n}(t) a_{n}^{*}=-\mathrm{i} \sum_{m} J_{n m} a_{m} a_{n}^{*} \times \\
& -\mathrm{i}\left|a_{n}\right|^{2} s \frac{f_{1}\left(\lambda_{n}\right) f_{2}\left(\zeta_{n}\right)}{\alpha^{2}}\left(\left(1+\frac{1}{2} s\right) f_{1}\left(\lambda_{n}\right) f_{2}^{3}\left(\zeta_{n}\right)-1\right) .
\end{aligned}
$$

The last term of the equation is purely imaginary so adding its complex conjugate the whole term vanishes. What remains is

$$
\dot{a}_{n}(t) a_{n}^{*}+\text { c.c. }=-\mathrm{i} \sum_{m} J_{n m} a_{m} a_{n}^{*}+\mathrm{i} \sum_{m} J_{n m} a_{m}^{*} a_{n} .
$$

The summation over $n$ yields the required result provided $J_{m n}=J_{n m}$. The second, energy conservation, condition is cumbersome, however, we observe its presence in all numerical simulations.

\subsection{Electronic ground state}

In many spectroscopy applications [18] it is necessary to propagate the electronic ground state alongside the excited state. Notice that the nonlinearities are present in the ground state so the analytical solution is not possible. However, the equations of motion can be easily obtained by taking all $a_{n}=0$, and for a specific oscillator (we drop indices) we get

$$
\begin{aligned}
& \dot{\lambda}=\lambda^{(i)}+\mathrm{i} \frac{f_{1}(\lambda) f_{2}(\zeta)}{\alpha \sqrt{2}}\left(f_{1}(\lambda) f_{2}^{3}(\zeta)-1\right), \\
& 2 \dot{r}=-\sin (\theta)\left[1-f_{1}(\lambda) f_{2}(\zeta)\left(2 f_{1}(\lambda) f_{2}^{3}(\zeta)-1\right)\right] \\
& \dot{\theta}=-(1+\operatorname{coth}(2 r) \cos (\theta)) \times \\
& \times\left[1+f_{1}(\lambda) f_{2}(\zeta)\left(2 f_{1}(\lambda) f_{2}^{3}(\zeta)-1\right)\right]
\end{aligned}
$$

These equations of motion are essentially for a free Morse oscillator and can be used to study squeezing and displacements of nuclear wave packet in this system in general. 


\section{Numerical demonstrations}

For numerical analysis of the model we use the Julia programming language [19]. We consider a simple model system of two sites characterized by Eqs. 16-19. The free parameters that can be varied are the anharmonicity parameter $\alpha$ of oscillators and the coupling strength parameter that we define using the reorganization energy, which for this case is $\Lambda=s^{2} /\left(2 \alpha^{2}\right)$. The last parameters are contained in the electronic system matrix $J$.

First, we consider an electronic system whose natural electronic frequency is higher than the vibrational frequency, i.e. $J_{m n}>\omega_{0} \equiv 1$ :

$$
J^{(A)}=\left(\begin{array}{ll}
1 & 2 \\
2 & 0
\end{array}\right)
$$

We keep its sites strongly coupled so that the pure electronic system is characterized by delocalized eigenvectors (given in columns)

$$
\phi=\left(\begin{array}{ll}
0.7882 & 0.6154 \\
0.6154 & -0.7882
\end{array}\right)
$$

for eigenvalues 2.562 and -1.562 , respectively. Asymmetry between sites is included for stability in the behaviour of the dynamics. Reorganization energies for two sites are the same, $\Lambda_{n}=0.1$. Initial conditions for the $a_{n}$ parameters are taken to correspond to the higher energy electronic eigenstate obtained by, e.g. optical excitation. Then the displacements $\lambda_{i}=0$, and squeezings $\theta_{i}=0$, $r_{i}=10^{-3}$ (for convergence). We label this system as system $\mathrm{A}$.

Figure 1 demonstrates the evolution of system wavefunction parameters as a function of time in the short time scale: $q_{i}=\sqrt{2} \lambda_{i}^{(\mathrm{r})}$ is the expectation value of the coordinate operator. We clearly observe that the electronic system is switched from the stationary exciton state at $t=0$ by the vibrational wave packet as $q_{n}$ deviates from zero. Since the system is anharmonic, squeezings are generated with $r_{n} \neq 0$. The system moves from the stationary state in an oscillatory way, i.e. electronic population deviations become larger in the second vibrational period and this would continue at longer times. For comparison we also show the result of a harmonic model, obtained by taking $\alpha \rightarrow 0$. Technically this is realized by using $\alpha=10^{-4}$.

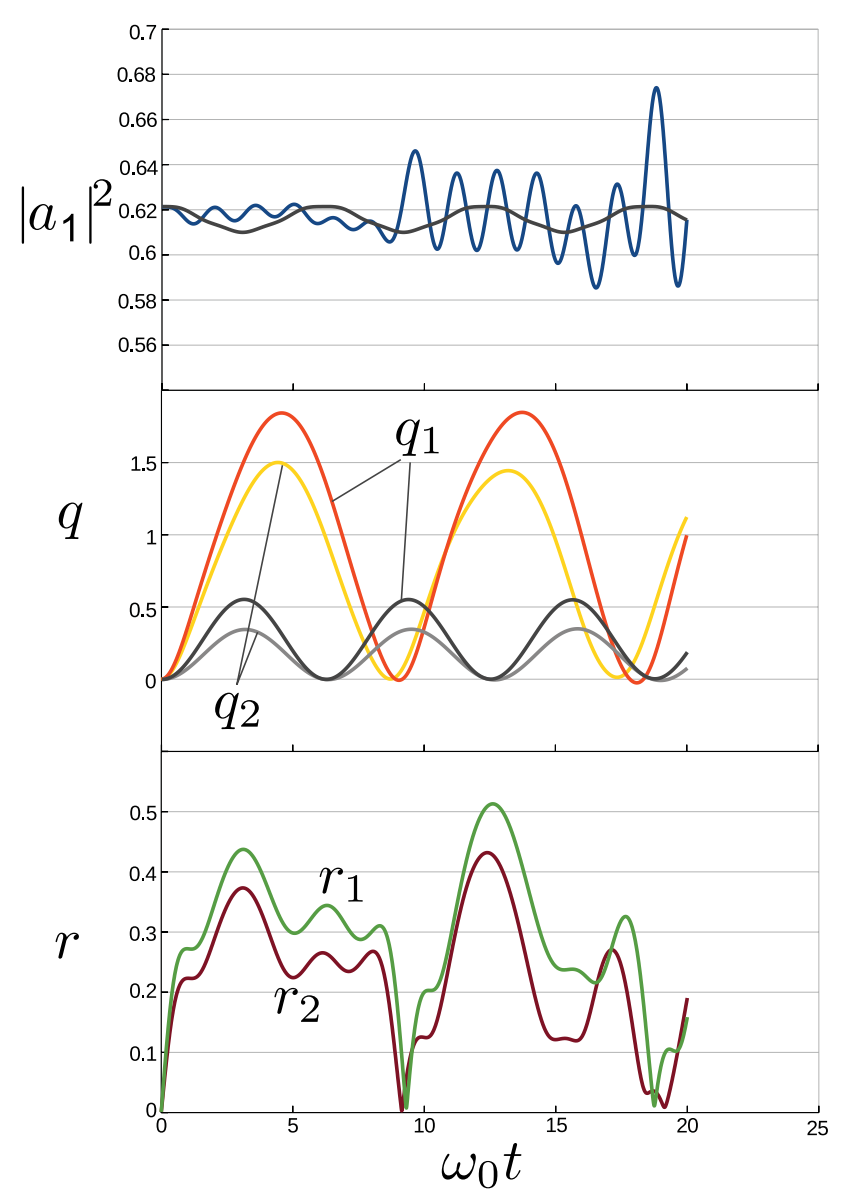

Fig. 1. Evolution of wavefunction parameters after excitation into the higher excitonic band of the model A. Black and grey (sine) curves are for the corresponding harmonic model $(\alpha \rightarrow 0)$. Colours (online) show the highly anharmonic case with $\alpha_{n}=0.5$.

In this case, the squeezing amplitudes do not evolve, the nuclear displacements are much smaller, while the electronic state varies in a reversible way. Hence, the anharmonicity introduces some kind of irreversibility on this short time scale.

Next we increase the electronic-vibrational interaction by taking the reorganization energies $\Lambda=1$ (we call this model model B). Figure 2 demonstrates the evolution of the system $\mathrm{B}$. In this case the excitation of sites leads to a very large deviation of vibrational coordinates from zero. Especially, the coordinate $q_{1}$ signifies almost dissociation, which is essentially allowed by the Morse potential. Squeezing also grows up dramatically. So the dissociation would lead to a highly squeezed wavepacket in the split wavefunction. The corresponding harmonic model (grey curves) does not allow dissociation, hence, the wave packet oscillations are much weaker. 


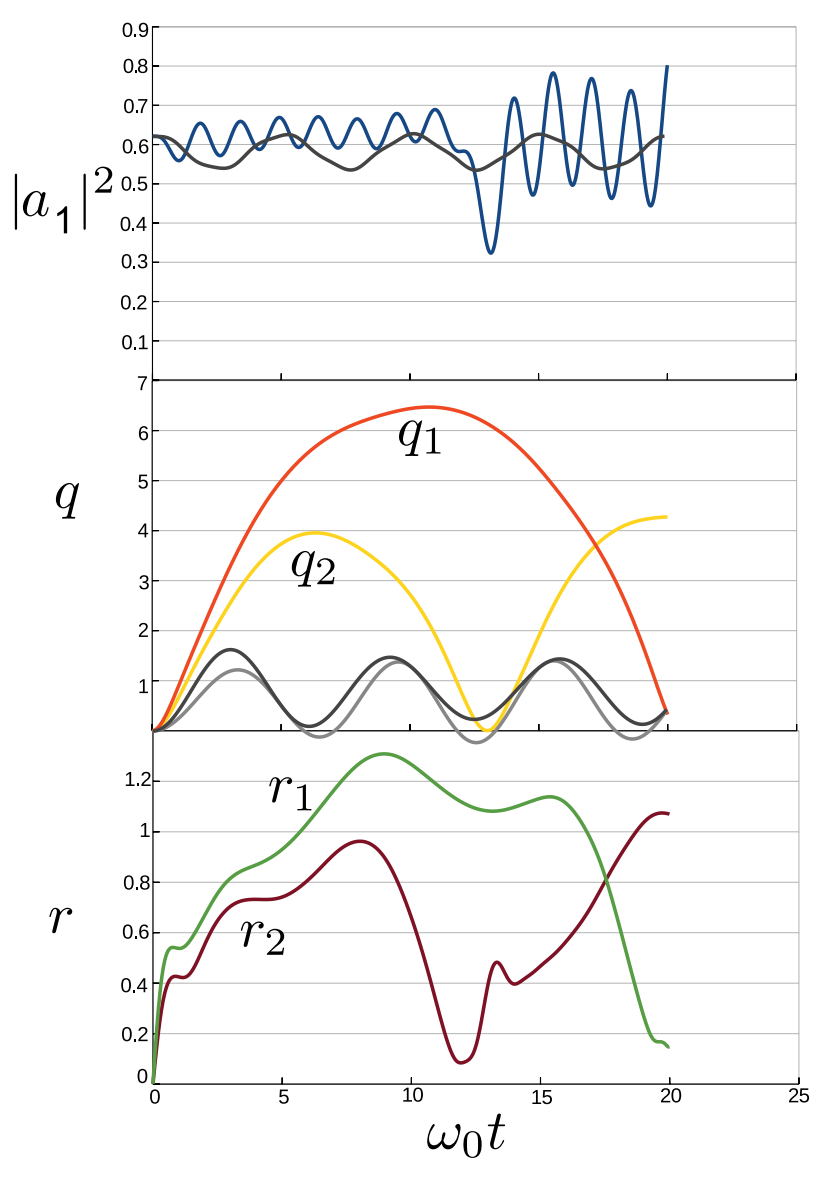

Fig. 2. Evolution of wavefunction parameters after excitation into the higher excitonic band of the model B. Black and grey (sine) curves are for the harmonic model. Colours (online) show the highly anharmonic case with $\alpha_{n}=0.5$.

So far we demonstrated that the anharmonicities lead to more complex irreversible dynamics on the short time scale. However, the more important question is how anharmonicities reshape the stationary states of the system. A whole set of eigenstates could be obtained by a 'brute force' approach by taking a specific set of orthogonal basis wavefunctions [14]. However, in this paper we rely on the Ansatz to find the lowest energy stationary state of the system. For this purpose we look for the minimum of the total energy, given by

$$
E=\langle\psi|\hat{H}| \psi\rangle
$$

Search for the minimum could be realized by a variational procedure, however, that would lead to a complicated nonlinear set of algebraic equations. Instead we use a simple Monte-Carlo numerical global energy minimization procedure, often de- noted by simulated annealing [20]. Starting from the above-used initial condition we perturb all parameters by small deviations randomly (the deviations are distributed according to the normal distribution) and at each iteration we choose a configuration that has a lower or equal energy compared to the previous case. Additionally of course we impose restrictions that the norm of the electronic amplitudes should be $a_{1}^{2}+a_{2}^{2}=1$, squeezing parameters $r_{i}>0$. Additionally to the systems $\mathrm{A}$ and $\mathrm{B}$, we also study the case of high vibrational frequencies by using $J \rightarrow 0.1 J^{(A)}$. This gives us four types of systems. A: $J=J^{(A)}, \Lambda_{n}=0.1, \mathrm{~B}: J=J^{(A)}$, $\Lambda_{n}=1, \mathrm{C}: J=0.1 J^{(A)}, \Lambda_{n}=0.1, \mathrm{D}: J=0.1 J^{(A)}, \Lambda_{n}=1$.

Figure 3 demonstrates a typical energy decay profile for the simulated annealing procedure. Thus, the minimum is reached in roughly 10000 steps. The same holds for all four systems A-D. The final energies are shown in Fig. 4. It shows

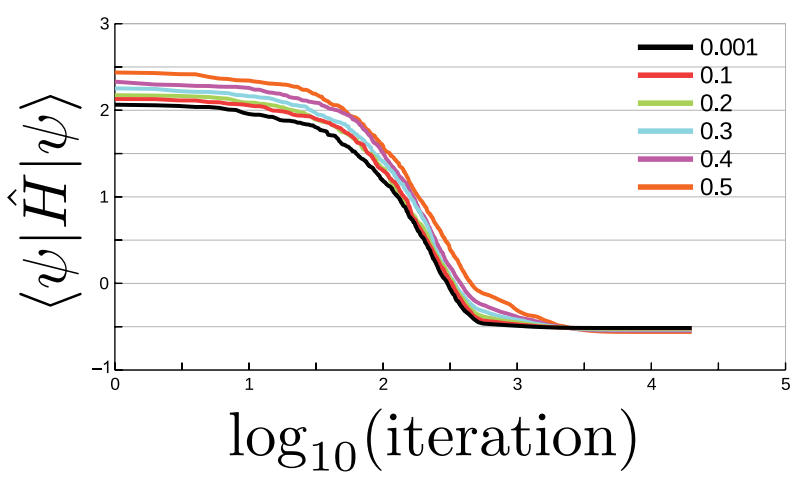

Fig. 3. Convergence of the total energy in the simulated annealing procedure for the system A depending on a different anharmonicity parameter $\alpha_{n}$ given in the inset.

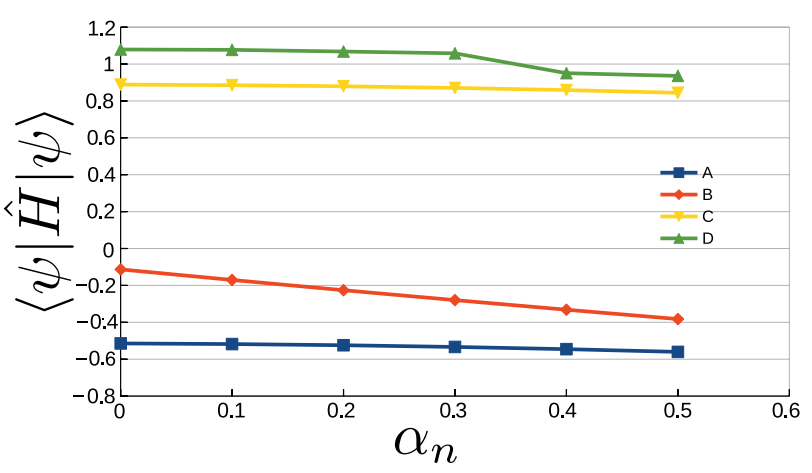

Fig. 4. Total energy after the simulated annealing procedure as a function of the anharmonicity parameter $\alpha_{n}$. A system type is given in the inset. 
that for the four system types larger anharmonicity leads to the decrease of the total energy. Apparently, the system has more flexibility and can better adapt to the electronic excitation.

Finally we present the configuration of the optimal state for the four systems A-D in Fig. 5. For the systems A-C the electronic populations $\left|a_{1}\right|^{2}$ grow up steadily as anharmonicity increases. At the same time the mean coordinate of the wavepacket grows up and the squeezing, while being small, grows up quadratically. Hence, the configuration (or the symmetry) of the optimal state does not change, while the localization grows up. We obtain a surprising result for the system $D$, where the symmetry of the wavefunction abruptly changes roughly at $\alpha_{n}=0.4$. It is interesting to note that the squeezing of the wavepacket does not change much, while vibrational wavepackets follow electronic populations.

\section{Discussion}

Molecular vibrations are never harmonic on a broad scale. Usually it is acceptable to follow a normal mode analysis of a molecule if we are interested in IR absorption, fluorescence or Raman spectra. However, nowadays the traditional two-dimensional infrared spectroscopy of molecular vibrations always demonstrates larger or smaller anharmonicities for all vibrations [21. 22]. Additionally, low temperature electronic hole burning or fluorescence line narrowing spectroscopy demonstrate ZPL, that has a measurable line width $[8,9,11]$. Moreover, this line width depends on the temperature. Such characteristics appear due to nonlinearities in the electron-vibrational interaction. Even without that, the thermal conduction and chemical reactions take place. Thus the Morse potential for molecular vibrations

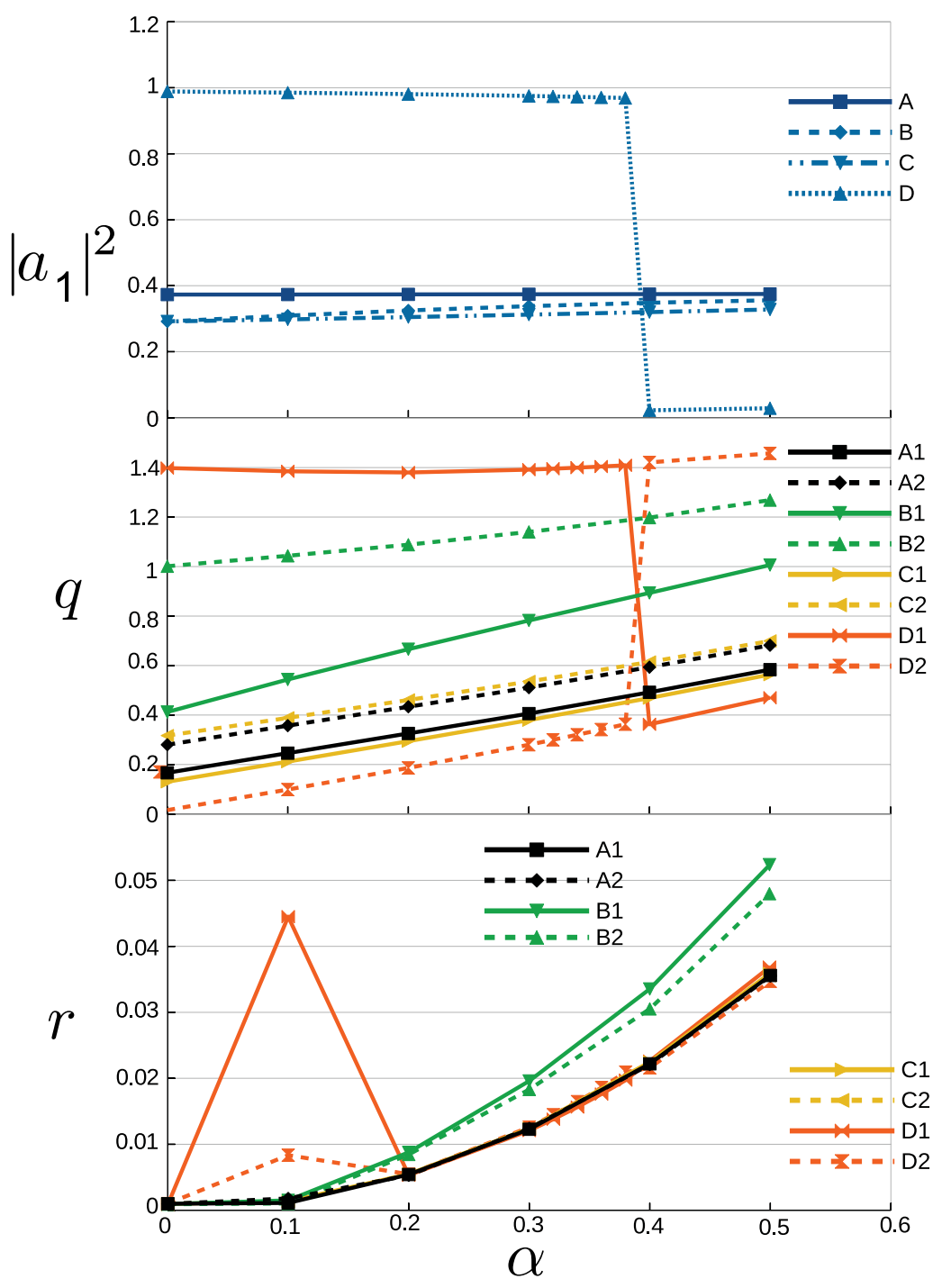

Fig. 5. Configuration of the optimized state as a function of anharmonicity. The models are indicated by letters $\mathrm{A}-\mathrm{D}$, while the parameter indices $n=1,2$ are labelled by numbers. 
seems much more natural than a harmonic oscillator model.

Our model formulation is very general. In principle all vibrational modes including phonons can be modelled in this way as Morse oscillators. In the harmonic case a convenient property is the spectral density of the vibrational manifold. It characterizes the spectral distribution of the electronic-vibrational couplings. With Morse oscillators, we can define the spectral density similar to the harmonic case as

$$
C_{n}^{\prime}(\omega)=\pi \omega^{2} \sum_{j} S_{n j} \delta\left(\omega-\omega_{j}\right)
$$

where $S_{n j}=\Lambda_{n j} \sqrt{2 \mathcal{D}_{i} \alpha_{i}^{2}}$ corresponds to the HuangRhys parameter, describing the coupling strength. Additionally we can define the spectral density of anharmonicities

$$
A_{n}(\omega)=\pi \sum_{j} \alpha_{j} \delta\left(\omega-\omega_{j}\right)
$$

Specific anharmonicity parameters $\alpha_{j}$ can be obtained for intra-molecular vibrations by quantum chemistry.

The four cases of parameters studied in this paper correspond to the typical situations: A, low frequency weakly coupled vibrational modes; $B$, low frequency strongly coupled vibrational modes; $\mathrm{C}$, a high frequency weakly coupled mode; $\mathrm{D}$, a high frequency strongly coupled mode. Surprisingly, the high frequency mode behaves slightly different compared to the low frequency case. The high frequency modes seem to have a larger effect on excitation, compared to the low frequency modes. The most remarkable effect of this is the strong localization of the system and additional phase transition - like change of configuration as nonlinearity $\alpha$ grows up.

A very interesting property of the model is the smooth tuning possibility of the anharmonicity parameter. The model interpolates between the harmonic modes and the modes prone to dissociation, or chemical reaction. Notice that in Fig. 2 the coordinate of the first oscillator grows up to very large values. In experimental conditions this would correspond to dissociation of an atomic entity or group. Consequently, van der Waals like interactions or specifically hydrogen bonding can be targeted using these equations of motion to reveal bistability, etc.
In addition to the dynamical approach given by time evolution of parameters, we presented an algorithm to look for a minimal energy of the system. This essentially leads to stationary polaronic states [23, 18. The system would not necessarily relax to these states in a specific experiment. It depends on the excited state lifetime. However, if the excited states live for a relatively long time so that the excited state relaxes to its minimum energy state, these states can be observed in, e. g. fluorescence spectroscopy.

To conclude, we developed a model where molecular vibrational degrees of freedom are represented by Morse oscillators. The model shows more flexibility and stability of the optimal lowest energy state compared to the harmonic model. The model also demonstrates the phase transition - like behaviour as a function of anharmonicity, thus implying the significance of an anharmonic nature of the vibrations.

\section{Appendix A \\ Main properties of displacement and squeezing operators}

Action of the displacement and squeezing operators on the ladder operators gives [24]

$$
\begin{aligned}
& \hat{D}^{\dagger}(\lambda) \hat{b} \hat{D}(\lambda)=\hat{b}+\lambda, \\
& \hat{D}^{\dagger}(\lambda) \hat{b}^{\dagger} \hat{D}(\lambda)=\hat{b}^{\dagger}+\lambda^{*},
\end{aligned}
$$

and

$$
\begin{aligned}
& \hat{S}^{\dagger}(\zeta) \hat{b} \hat{S}(\zeta)=\hat{b} \cosh (r)-b \mathrm{e}^{\mathrm{i} \theta} \sinh (r), \\
& \hat{S}^{\dagger}(\zeta) \hat{b}^{\dagger} \hat{S}(\zeta)=\hat{b}^{\dagger} \cosh (r)-\hat{b}^{-\mathrm{i} \theta} \sinh (r)
\end{aligned}
$$

where we used $\zeta=r \exp (\mathrm{i} \theta)$. Then we can show that

$$
\begin{aligned}
& \hat{S}^{\dagger}(\zeta) \hat{D}^{\dagger}(\lambda)\left(\hat{b}^{\dagger}+\hat{b}\right)^{m} \hat{D}(\lambda) \hat{S}(\zeta) \\
& =\left(\hat{b}^{\dagger} \xi^{*}+\hat{b} \xi+2 \Re(\lambda)\right)^{m}, \\
& \hat{S}^{\dagger}(\zeta) \hat{D}^{\dagger}(\lambda)\left(\hat{b}^{\dagger}-\hat{b}\right)^{m} \hat{D}(\lambda) \hat{S}(\zeta) \\
& =\left(b^{\dagger} \psi^{*}+\hat{b} \psi+2 \Re(\lambda)\right)^{m} .
\end{aligned}
$$

Here

$$
\begin{aligned}
& \xi=\cosh (r)-\mathrm{e}^{-\mathrm{i} \theta} \sinh (r), \\
& \psi=\cosh (r)+\mathrm{e}^{-\mathrm{i} \theta} \sinh (r) .
\end{aligned}
$$


Consequently we can calculate

$$
\begin{aligned}
& \left\langle 0_{v}\left|\hat{S}^{\dagger}(\zeta) \hat{D}^{\dagger}(\lambda) \exp (-\alpha \hat{x}) \hat{D}(\lambda) S(\zeta)\right| 0_{v}\right\rangle \\
& =\left\langle 0_{v} \exp \left(-\frac{\alpha}{\sqrt{2 \omega}}\left(\hat{b}^{\dagger} \xi^{*}+\hat{b} \xi+2 \Re(\lambda)\right)\right) \mid 0_{v}\right\rangle \\
& =\exp \left(-\frac{\alpha}{\sqrt{2 \omega}}\left(\lambda+\lambda^{*}\right)\right) \exp \left(\frac{\alpha^{2} \xi^{*} \xi}{4 \omega}\right) .
\end{aligned}
$$

For this purpose we used the Baker-CampbellHausdorff formula 25

$$
\mathrm{e}^{X+Y}=\mathrm{e}^{X} \mathrm{e}^{Y} \mathrm{e}^{-\frac{1}{2}[X, Y]} \ldots
$$

Additional useful relations are the expectation values for the potential energy of a harmonic oscillator

$$
\omega^{2} \frac{\hat{x}^{2}}{2}=\omega \frac{\left(\hat{b}^{+}+\hat{b}\right)^{2}}{4} \rightarrow \omega \frac{|\xi|^{2}+\left(\lambda+\lambda^{*}\right)^{2}}{4}
$$

and the general kinetic energy

$$
\frac{p^{2}}{2}=\frac{\hat{\dot{x}}^{2}}{2}=-\frac{\omega\left(\hat{b}^{+}-\hat{b}\right)^{2}}{4} \rightarrow \omega \frac{|\psi|^{2}-\left(\lambda^{*}-\lambda\right)^{2}}{4} .
$$

The quantities that enter the expressions are

$$
\begin{aligned}
& |\xi|^{2}=\cosh (2 r)-\sinh (2 r) \cos (\theta), \\
& |\psi|^{2}=\cosh (2 r)+\sinh (2 r) \cos (\theta) .
\end{aligned}
$$

\section{Appendix B}

\section{Normal ordered forms of operators, auxiliary functions and derivatives}

For the squeezing operator we use the factorization expression [25]

$$
\begin{aligned}
& \exp \left(\frac{\zeta^{*} \hat{b}^{2}-\zeta \hat{b}^{\dagger 2}}{2}\right)=\exp \left(\frac{-y \hat{b}^{\dagger 2}}{2}\right) \times \\
& \times\left(1-|y|^{2}\right) \exp \left(\frac{\hat{b}^{\dagger} \hat{b}+1 / 2}{2}\right) \exp \left(\frac{y^{*} \hat{b}^{2}}{2}\right) .
\end{aligned}
$$

Here

$$
y=\mathrm{e}^{\mathrm{i} \theta} \tanh r .
$$

For the displacement operator we have

$$
\exp \left(\lambda \hat{b}^{\dagger}-\lambda^{*} \hat{b}\right)=\exp \left(\lambda \hat{b}^{\dagger}\right) \exp \left(-\lambda^{*} \hat{b}\right) \exp \left(-\frac{\lambda^{*} \lambda}{2}\right) \text {. }
$$

Hence we have well defined time derivatives:

$$
\begin{aligned}
& \frac{\mathrm{d}}{\mathrm{d} t} \hat{S}(\zeta)=\frac{-\dot{y} \hat{b}^{\dagger 2}}{2} \hat{S}(\zeta) \\
& -\frac{\dot{y} y^{*}+y \dot{y}^{*}}{1-|y|^{2}} \hat{S}(\zeta)+\hat{S}(\zeta) \frac{\dot{y}^{*} \hat{b}^{2}}{2}, \\
& \frac{\mathrm{d}}{\mathrm{d} t} \hat{D}(\lambda)=\dot{\lambda} \hat{b}^{\dagger} \hat{D}(\lambda)-\dot{\lambda}^{*} \hat{D}(\lambda) \hat{b}-\frac{\lambda^{*} \lambda+\lambda^{*} \dot{\lambda}}{2} \hat{D}(\lambda) .
\end{aligned}
$$

To simplify the final equations of motion (Eqs. 12-15) we define

$$
\begin{aligned}
& f_{1}(\lambda)=\exp \left(-\frac{2 \alpha}{\sqrt{2 \omega}} \lambda^{(\mathrm{r})}\right) \\
& f_{2}(\zeta) \equiv f_{2}(r, \theta)=\exp \left(\frac{\alpha^{2}}{4 \omega}(\cosh (2 r)\right. \\
& -\sinh (2 r) \cos (\theta))) .
\end{aligned}
$$

Then derivatives with respect to parameters are as follows:

$$
\begin{aligned}
& \frac{\partial}{\partial \lambda^{*}} f_{1}(\lambda)=-\frac{\alpha}{\sqrt{2 \omega}} f_{1}(\lambda) \\
& \frac{\partial}{\partial \theta} f_{2}(\zeta)=\frac{\alpha^{2}}{4 \omega} \sinh (2 r) \sin (\theta) f_{2}(\zeta) \\
& \frac{\partial}{\partial r} f_{2}(\zeta)=\frac{\alpha^{2}}{4 \omega}(2 \sinh (2 r)-2 \cosh (2 r) \cos (\theta)) f_{2}(\zeta)
\end{aligned}
$$

Additional important derivatives originate due to the factorization of the squeezing operator. As $y=\mathrm{e}^{\mathrm{i} \theta} \tanh r$ we have

$$
\dot{y}=\mathrm{i} \theta \dot{\mathrm{e}^{\mathrm{i} \theta}} \tanh r+\frac{\mathrm{e}^{\mathrm{i} \theta} \dot{r}}{\cosh ^{2} r},
$$

and the quantity entering the kinetic part of the Lagrangian yields

$$
\dot{y} \mathrm{e}^{-\mathrm{i} \theta_{n}}-\dot{y}^{*} \mathrm{e}^{\mathrm{i} \theta_{n}}=2 \mathrm{i} \dot{\theta} \tanh r .
$$

Finally

$$
\begin{aligned}
& \frac{\partial}{\partial \theta}|\psi|^{2}=-\sinh (2 r) \sin (\theta), \\
& \frac{\partial}{\partial r}|\psi|^{2}=2 \sinh (2 r)+2 \cosh (2 r) \cos (\theta),
\end{aligned}
$$




$$
\begin{aligned}
& \frac{\partial}{\partial r}|\xi|^{2}=\sinh (2 r) \sin (\theta), \\
& \frac{\partial}{\partial r}|\xi|^{2}=2 \sinh (2 r)-2 \cosh (2 r) \cos (\theta) .
\end{aligned}
$$

\section{Appendix C}

\section{Different parts of the Lagrangian}

The potential energy term of a single Morse oscillator

$$
\begin{aligned}
& V(\hat{x})=\mathcal{D}(1-\exp (-\alpha \hat{x}))^{2}= \\
& \mathcal{D}(1-2 \exp (-\alpha \hat{x})+\exp (-2 \alpha \hat{x}))
\end{aligned}
$$

Using the appendix A, we can immediately write

$$
\begin{aligned}
& \left\langle\psi\left|V_{i}\left(\hat{x}_{i}\right)\right| \psi\right\rangle=\mathcal{D}_{i}\left(1-2 f_{1}\left(\lambda_{i}\right) f_{2}\left(\zeta_{i}\right)\right. \\
& \left.+f_{1}^{2}\left(\lambda_{i}\right) f_{2}^{4}\left(\zeta_{i}\right)\right)
\end{aligned}
$$

where auxiliary functions are defined according to Eqs. 51 and 52. Next, the kinetic energy term of the Morse oscillator

$$
\begin{aligned}
& \left\langle\psi\left|\sum_{i} \hat{K}\left(\hat{x}_{i}\right)\right| \psi\right\rangle=\sum_{i} \frac{\omega_{i}}{4}\left(\cosh \left(2 r_{i}\right)\right. \\
& \left.+\sinh \left(2 r_{i}\right) \cos \left(\theta_{i}\right)-\left(\lambda_{i}^{*}-\lambda_{i}\right)^{2}\right),
\end{aligned}
$$

and the electronic-vibrational interaction term

$$
\begin{aligned}
& |n\rangle\langle n| \sum_{i} \mathcal{D}_{i} s_{i n}\left(\left(2+s_{i n}\right) \mathrm{e}^{-2 \alpha_{i} \hat{x}_{i}}-2 \mathrm{e}^{-\alpha_{i} \hat{x}_{i}}\right) \rightarrow \\
& \rightarrow a_{n}^{*} a_{n} \sum_{i} \mathcal{D}_{i} s_{i n}\left(\left(2+s_{i n}\right) f_{1}^{2}\left(\lambda_{i}^{(r)}\right) f_{2}^{4}\left(r_{i}, \theta_{i}\right)\right. \\
& \left.-2 f_{1}\left(\lambda_{i}^{(\mathrm{r})}\right) f_{2}\left(r_{i}, \theta_{i}\right)\right) .
\end{aligned}
$$

The remaining kinetic part is essentially the most complicated requiring normal ordering of all operators:

$$
\begin{aligned}
& \frac{\mathrm{i}}{2}\left(\left\langle\psi \mid \frac{\mathrm{d}}{\mathrm{d} t} \psi\right\rangle-\left\langle\frac{\mathrm{d}}{\mathrm{d} t} \psi \mid \psi\right\rangle\right)= \\
& =\frac{\mathrm{i}}{2} \sum_{n}\left(a_{n}^{*} \dot{a}_{n}-a_{n} \dot{a}_{n}^{*}\right) \\
& +\frac{1}{2} \sum_{i}\left(\mathrm{i} \lambda_{i}^{*} \dot{\lambda}_{i}-\mathrm{i} \dot{\lambda}_{i}^{*} \lambda_{i}-\dot{\theta}_{i} \sinh ^{2}\left(r_{i}\right)\right) .
\end{aligned}
$$

\section{References}

[1] M. Pope and C.E. Swenberg, Electronic Processes in Organic Crystals and Polymers, 2nd ed. (Oxford University Press, Oxford and New York, 1999).

[2] M.C. Petty, Organic and Molecular Electronics: From Principles to Practice (Wiley, 2019).

[3] R.E. Blankenship, Molecular Mechanisms of Photosynthesis, 2nd ed. (Wiley Blackwell, Oxford, UK; Chichester, UK; Hoboken, USA, 2014).

[4] H. van Amerongen, L. Valkunas, and R. van Grondelle, Photosynthetic Excitons (World Scientific, Singapore, New Jersey, London, Hong Kong, 2006).

[5] A. Davydov, Theory of Molecular Excitons (Springer, 1971).

[6] L. Valkunas, D. Abramavicius, and T. Mančal, Molecular Excitation Dynamics and Relaxation (Wiley-VCH Verlag GmbH \& Co. KGaA, Weinheim, Germany, 2013).

[7] S.S. Mukamel, Principles of Nonlinear Optical Spectroscopy (Oxford University Press, New York, 1995).

[8] R. Jankowiak, M. Reppert, V. Zazubovich, J. Pieper, and T. Reinot, Site selective and single complex laser-based spectroscopes: A window on excited state electronic structure, excitation energy transfer, and electron-phonon coupling of selected photosynthetic complexes, Chem. Rev. 111, 4546-4598 (2011).

[9] O. Rancova, R. Jankowiak, and D. Abramavicius, Probing environment fluctuations by two-dimensional electronic spectroscopy of molecular systems at temperatures below $5 \mathrm{~K}$, J. Chem. Phys. 142(21), 212428 (2015).

[10]D. Abramavicius, V. Chorošajev, and L. Valkunas, Tracing feed-back driven exciton dynamics in molecular aggregates, Phys. Chem. Chem. Phys. 20, 21225-21240 (2018).

[11]Zero-Phonon Lines and Spectral Hole Burning in Spectroscopy and Photochemistry, ed. K.H. Olev Sild (Springer-Verlag, 1988).

[12]A. Galestian Pour, C.N. Lincoln, V. Perlik, F. Šanda, and J. Hauer, Anharmonic vibrational effects in linear and two-dimensional 
electronic spectra, Phys. Chem. Chem. Phys. 19, 24752-24760 (2017).

[13]M. Dinpajooh and D.V. Matyushov, NonGaussian lineshapes and dynamics of timeresolved linear and nonlinear (correlation) spectra, J. Phys. Chem. B 118(28), 7925-7936 (2014).

[14]A. Anda, D. Abramavičius, and T. Hansen, Two-dimensional electronic spectroscopy of anharmonic molecular potentials, Phys. Chem. Chem. Phys. 20, 1642-1652 (2018).

[15]V. Chorošajev, T. Marčiulionis, and D. Abramavicius, Temporal dynamics of excitonic states with nonlinear electron-vibrational coupling, J. Chem. Phys. 147(7), 074114 (2017).

[16]V. Butkus, L. Valkunas, and D. Abramavicius, Vibronic phenomena and exciton-vibrational interference in two-dimensional spectra of molecular aggregates, J. Chem. Phys. 140(3), 034306 (2014).

[17]L. Chen, M. Gelin, and Y.Zhao, Dynamics of the spin-boson model: A comparison of the multiple Davydov $\mathrm{D}_{1}, \mathrm{D}_{1.5}, \mathrm{D}_{2}$ Ansätze, Chem. Phys. [in press, corrected proof, 2018], https:// doi.org/10.1016/j.chemphys.2018.08.041

[18]V. Chorošajev, O. Rancova, and D. Abramavicius, Polaronic effects at finite temperatures in the B850 ring of the LH2 complex, Phys. Chem. Chem. Phys. 18, 7966 (2016).

[19]J. Bezanson, A. Edelman, S. Karpinski, and V. Shah, Julia: A fresh approach to numerical computing, SIAM Rev. 59(1), 65-98 (2017).

[20]W.H. Press, S.A. Teukolsky, W.T. Vetterling, and B.P. Flannery, Numerical Recipes: The Art of Scientific Computing, 3rd ed. (Cambridge University Press, 2007).

[21]P. Hamm, M. Lim, and R.M. Hochstrasser, Structure of the amide I band of peptides measured by femtosecond nonlinear-infrared spectroscopy, J. Phys. Chem. B 102(31), 6123-6138 (1998).

[22]M. Cho, Two-Dimensional Optical Spectroscopy, 1st ed. (CRC Press, 2009).

[23]A. Gelzinis, D. Abramavicius, and L. Valkunas, Non-Markovian effects in time-resolved fluorescence spectrum of molecular aggregates: Tracing polaron formation, Phys. Rev. B 84, 245430 (2011).

[24]J.-P. Gazeau, Coherent States in Quantum Physics (Wiley-VCH, 2009).

[25]D.R. Truax, Baker-Campbell-Hausdorff relations and unitarity of $\mathrm{SU}(2)$ and $\mathrm{SU}(1,1)$ squeeze operators, Phys. Rev. D 31, 1988-1991 (1985).

\title{
SUŽADINIMO DINAMIKA DVIEJŲ LYGIŲ KVANTINĖJE SISTEMOJE, SĄVEIKAUJANČIOJE SU MORSO VIRPESIAIS
}

\author{
D. Abramavičius, T. Marčiulionis \\ Vilniaus universiteto Chemines fizikos institutas, Vilnius, Lietuva
}

\section{Santrauka}

Molekulinio agregato, sąveikaujančio su Morso tipo virpesiais, sužadintų būsenų kvantinè dinamika modeliuojama naudojant nuo laiko priklausantị variacinị metodą. Gautos sužadintos būsenos elektroninių amplitudžių, virpesinių bangų paketų poslinkių ir susispaudimų bendros judejimo lygtys.
Kompiuterinis modeliavimas parodo, kad virpesiuc anharmoniškumas įneša judejjimo negrižtamumą labai trumpų laikų skaleje ir papildomą elektroninio sužadinimo lokalizaciją. Be to, žemiausios energijos sužadintos būsenos simetrija dèl anharmoniškumo gali pakisti iš esmès. 